\title{
Evidence of Bose-Einstein Condensation in an Atomic Gas with Attractive Interactions
}

\author{
C. C. Bradley, C. A. Sackett, J. J. Tollett, and R. G. Hulet \\ Physics Department and Rice Quantum Institute, Rice University, Houston, Texas 77251-1892
}

(Received 25 July 1995)

\begin{abstract}
Evidence for Bose-Einstein condensation of a gas of spin-polarized ${ }^{7} \mathrm{Li}$ atoms is reported. Atoms confined to a permanent-magnet trap are laser cooled to $200 \mu \mathrm{K}$ and are then evaporatively cooled to lower temperatures. Phase-space densities consistent with quantum degeneracy are measured for temperatures in the range of 100 to $400 \mathrm{nK}$. At these high phase-space densities, diffraction of a probe laser beam is observed. Modeling shows that this diffraction is a sensitive indicator of the presence of a spatially localized condensate. Although measurements of the number of condensate atoms have not been performed, the measured phase-space densities are consistent with a majority of the atoms being in the condensate, for total trap numbers as high as $2 \times 10^{5}$ atoms. For ${ }^{7} \mathrm{Li}$, the spin-triplet $s$-wave scattering length is known to be negative, corresponding to an attractive interatomic interaction. Previously, Bose-Einstein condensation was predicted not to occur in such a system.
\end{abstract}

PACS numbers: 03.75.Fi, 05.30.Jp, 32.80.Pj

Bose-Einstein condensation (BEC) of an ideal gas, predicted more than 70 years ago [1], is the paradigm of quantum statistical phase transitions. This phase transition is manifested by an abrupt growth in the population of the ground state of the potential confining the gas. BEC occurs when the mean particle separation is comparable to the thermal de Broglie wavelength $\Lambda$. Although BEC-like phase transitions have been observed in liquid $\mathrm{He}$ and in excitonic systems [2], BEC of a weakly interacting gas has been a long sought, but elusive goal [3]. Recently this goal was realized in a rarified gas of spin-polarized, ultracold ${ }^{87} \mathrm{Rb}$ atoms [4]. Because of the extraordinarily low temperatures that can be achieved in atomic gases, BEC can be studied in the low density regime where interatomic distances are much greater than the distance scale of atomatom interactions. In this regime, two-body interactions take a simple form, and three-body interactions can be neglected. It is hoped that investigations of these relatively simple quantum degenerate systems will enable a better understanding of more complex Bose systems, such as superconductors, where particle interactions are significant.

It is possible that even the weak interactions between atoms of an ultracold gas can result in behavior in the quantum degenerate regime that departs significantly from that of an ideal gas. At low temperatures and densities, only two-body $s$-wave scattering is important. If the temperature is so low that the magnitude of the $s$-wave scattering length $a$ is much less than $\Lambda$, then the exact shape of the atom-atom interaction potential is unimportant [5]. For a purely repulsive potential, $a$ is positive, while for a purely attractive potential that supports no bound states, $a$ is negative. The spin-triplet interaction potential between alkali-metal atoms supports bound states. In such a case, $a$ can be either positive or negative depending on the proximity of the least bound state to the dissociation limit [6]. From recent analysis of the photoassociative spectrum of ultracold ${ }^{85} \mathrm{Rb}$, it is believed that $a$ is positive for ${ }^{87} \mathrm{Rb}$ [7]. The scattering length is accurately known for the triplet potential of ${ }^{7} \mathrm{Li}$; direct two-photon photoassociation spectroscopy of the least bound triplet ground state of ${ }^{7} \mathbf{L i}_{2}$ combined with analysis of previous ${ }^{7} \mathrm{Li}_{2}$ spectra [8] give $a=(-27.3 \pm$ 0.8) $a_{0}$, where $a_{0}$ is the Bohr radius [9].

The condensate wave function can be described by a nonlinear Schrödinger equation, in which the interatomic interactions are represented by a mean-field pseudopotential $[5,10]$. For a spatially homogeneous gas with $a>0$, there are stable solutions to this equation, and, for low densities, the properties of the gas are given by an expansion in the small parameter $\left(n a^{3}\right)^{1 / 2}$, where $n$ is the density [11]. The situation is drastically different for $a<0$, because the gas is not mechanically stable against collapse [10]. It was predicted that for an atom with negative $a$ BEC would be preempted by a first-order gas-liquid or gas-solid phase transition [12]. However, we and others suggested that the situation may be quite different in the spatially inhomogeneous environment of an atom trap. In a potential, it is possible for the zero-point energy to exceed the attractive interaction for a limited number of condensate atoms, stabilizing the gas against collapse. This suggestion has been developed formally by solving the nonlinear Schrödinger equation with a Hamiltonian that includes the confining potential [13]. However, the solution is valid only for temperature $T=0$, and the theory does not address the dynamical issue of whether a condensate can form for $a<0$. Consequently, the question of whether a weakly interacting Bose gas with attractive interactions could undergo BEC has remained open. In this Letter, we report evidence for BEC of a gas of spin-polarized ${ }^{7} \mathrm{Li}$.

The apparatus was described in detail in a previous publication [14]. The atoms are confined in a magnetic trap that is produced by six permanent magnet cylinders. The magnets are arranged to produce a minimum at the trap center near which the field strength varies quadratically. The minimum is offset by a uniform bias field of $823 \mathrm{G}$ along the $z$ axis. This field distribution was used because 
the bias field prevents the loss of atoms due to nonadiabatic spin-flip transitions that can occur at the center of magnetic traps that do not have a bias field $[15,16]$. The trap oscillation frequencies of $\nu_{z}=117 \pm 6 \mathrm{~Hz}$ and $\nu_{x, y}=$ $163 \pm 15 \mathrm{~Hz}$ were determined by a combination of spectroscopic observations, direct magnetic field measurement, and the observed spatial asymmetry of the trapped atom cloud [17]. These values determine the critical number for the BEC phase transition to be $N_{c}=3.5 \times 10^{6}(T / \mu \mathrm{K})^{3}$ [18]. The trap is loaded from a laser-slowed atomic beam that passes through the center of the trap. Three pairs of counterpropagating laser beams, tuned to near the $2 S_{1 / 2}, F=2, m_{F}=2 \leftrightarrow 2 P_{3 / 2}, F=3, m_{F}=3$ transition frequency at the bias magnetic field $(\lambda=671 \mathrm{~nm}$ wavelength), are directed along three axes passing through the trap center. Through Doppler cooling, these beams provide the dissipation necessary for trap loading. Each of the six trap loading beams is linearly polarized and has an intensity of $17 \mathrm{~mW} / \mathrm{cm}^{2}$. Loading typically results in $\sim 2 \times 10^{8}$ trapped atoms. Upon completion of loading, the intensity of the trap loading beams is reduced by a factor of 50 to cool the atoms to $\sim 200 \mu \mathrm{K}$, which is near the Doppler cooling limit of $140 \mu \mathrm{K}$. The peak density after Doppler cooling is $\sim 7 \times 10^{10} \mathrm{~cm}^{-3}$. The lifetime for the atoms to remain trapped, limited presumably by collisions with background gas atoms, exceeds $10 \mathrm{~min}$. By extrapolation, the background gas pressure is estimated to be near $10^{-12}$ Torr, and is achieved using both an ion and a titanium sublimation pump on a chamber with only a narrow, conduction-limiting tube connecting it to the slowatom source [14].

The atoms are cooled further by evaporation, a process whereby the hottest atoms are selectively removed and the remainder are allowed to rethermalize via elastic two-body collisions [19]. This technique was demonstrated several years ago using magnetically trapped spin-polarized $\mathrm{H}[20]$, and recently using laser cooled alkali-metal atoms [16,21]. Given the initial temperature, density, and elastic scattering cross section of $5.0 \times 10^{-13} \mathrm{~cm}^{2}$ [9], the number of rethermalizing collisions per trap lifetime is $\sim 1000$, which is sufficient for efficient evaporative cooling. A microwave field is tuned to selectively drive the electron spinflip transition for atoms at a particular value of magnetic field, and therefore at a unique trap radius $[16,22]$. The microwave field is produced by a frequency synthesizer and coupled to a loop antenna inside the vacuum chamber. The frequency vs time trajectory that optimizes phase-space density was determined by modeling the time-dependent energy distribution [23], including losses due to background gas collisions and two-body dipolar relaxation collisions [24]. The calculated trajectory for our initial conditions crosses the BEC phase transition point at $T=$ $300 \mathrm{nK}$ and $N=1 \times 10^{5}$, after $\sim 5$ min of evaporation.

An absorption probe is used to measure the number and temperature of the trapped atoms. This probe laser beam is directed through the atom cloud, and its nearfield absorption field is imaged by a $12 \mathrm{~cm}$ focal length compound lens positioned outside the chamber at a distance of $17 \mathrm{~cm}$ from the atoms. The image is recorded by a slow-scan charge-coupled-device (CCD) camera. An aperture stop of $3 \mathrm{~cm}$ diameter is located at the lens to reduce spherical aberration. The probe is a collimated Gaussian beam with a waist ( $e^{-2}$ radius) of $1.5 \mathrm{~mm}$ and a power of $1 \mathrm{~mW}$. The probe frequency is typically detuned by $12-18 \mathrm{MHz}$ (2-3 natural linewidths) below the atomic resonance frequency at the bottom of the trap, so that the probe absorption is relatively weak and also so that differences in Zeeman shifts among the atoms are negligible. The probe beam propagates along the $x=y=z$ body-diagonal axis of the trap and is linearly polarized perpendicular to the trap bias field. Because the probe polarization does not decompose into pure $\sigma^{+}$ polarization in the trap coordinates, not all of the light can be absorbed by the atoms. Given that the probe propagates at an angle of $54.7^{\circ}$ with respect to the bias field, a maximum of $75 \%$ of the probe beam intensity is absorbable. In the following discussion, probe absorption is expressed relative to this maximum value.

An image is recorded by pulsing on the probe for $30 \mu \mathrm{s}$. Probing heats the sample, so the trap must be reloaded and the evaporative cooling cycle repeated for each measurement. By comparing the image to one taken with no atoms in the trap, the spatial absorption profile is obtained. The total number of missing photons, that is, the total absorption, provides a measure of $N$. For low $N$, the uncertainty is $2 \times 10^{4}$ due to the intensity noise of the probe. For larger numbers, the uncertainty is $20 \%$ due to the $1.5 \mathrm{MHz}$ uncertainty in the probe detuning. The temperature of the atoms is determined by the spatial size of the absorption image. Because the trap spring constants are asymmetric, the image has an elliptical shape, so a profile of absorption vs radius is obtained by scaling the axes of the ellipse and performing an angular average about the center of the cloud. Assuming a Boltzmann energy distribution, the radius at which the optical density of the cloud has fallen to $e^{-1}$ of its peak value determines the temperature, with an accuracy of $25 \%$. In addition, the peak optical density of the cloud (accurate to $5 \%$ ) combined with the $e^{-1}$ radius provides a second value for $N$; these values typically agree to within their uncertainties. The peak optical density, the $e^{-1}$ radius, and the trap oscillation frequencies can also be used to calculate the critical parameter $N / N_{c}$ with an accuracy of $50 \%$.

Figure 1(a) shows an image in which the atom cloud has an $e^{-1}$ radius of $36 \mu \mathrm{m}$ in the trap $x-y$ direction, and a peak absorption of $22 \%$. These values correspond to $1.2 \times 10^{5}$ atoms with a temperature of $590 \mathrm{nK}$, giving $N / N_{c}=0.2$. Figure 1 (b) shows the result of a separate load-evaporate cycle, giving $N / N_{c}>1$, in which a distinct ringlike halo surrounding the absorption peak is observed. Figure 2(a) shows a cross-sectional profile, and Fig. 2(b) shows the angular average of these data. The halo is evident as a region of negative absorption surrounding the peak. All of the 16 images of clouds with 


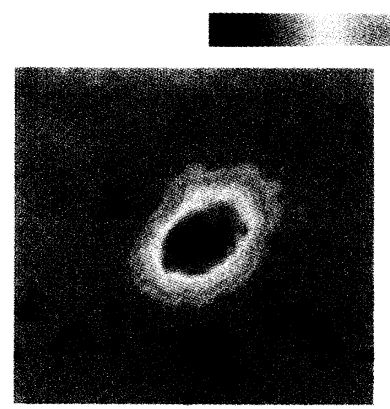

(a)

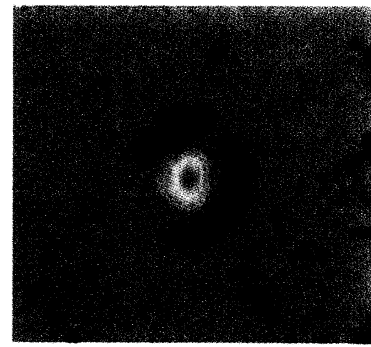

(b)
FIG. 1 (color). False-color images of trapped atom clouds resulting from different load-evaporate cycles. Images are obtained by passing a probe laser beam through the atom cloud and imaging its near-field absorption profile on a CCD camera. These images are then subtracted from a background image taken without atoms present. The spatial scale corresponds to $250 \mu \mathrm{m}$ along each axis at the location of the atoms. In these images, the diagonal extending from the lower right corner to the upper left corner is aligned with the trap $x-y$ direction. The color scale above the images maps the colors to probe absorption. The scale varies linearly from $-15 \%$ (violet) to $20 \%$ (red) absorption, where negative values indicate areas that are brighter in the presence of atoms. (a) corresponds to $1.2 \times 10^{5}$ atoms at $590 \mathrm{nK}$, and (b) to the nominal values of $2 \times 10^{4}$ atoms at $100 \mathrm{nK}$. The halo surrounding the absorption peak visible in (b) is attributed to diffraction from the spatially localized Bose-Einstein condensate.

$N / N_{c}>1.5$ exhibit a halo, while none of the images with $N / N_{c}<0.7$ do. The remaining 5 images are consistent with either $N / N_{c}<1$ or $N / N_{c}>1$, given the measurement uncertainty. Quantum degenerate conditions were attained and halos observed for temperatures as high as $400 \mathrm{nK}$, and total numbers as great as $2 \times 10^{5}$ atoms.

We interpret these halos as being due to diffraction of the probe beam from the small Bose-Einstein condensate. The probe beam diffracts from atom clouds at any temperature, but, as long as the entire diffraction pattern is captured by the lens, the near-field image recorded by the camera will be an accurate depiction of the absorption profile at the atoms and will appear smooth and nearly Gaussian. The observation of a diffraction pattern in an image indicates that not all of the probe light is captured by the lens. For our imaging system, this can only occur if the scatterer is smaller than or comparable to the $3 \mu \mathrm{m}$ ground state of the trap. By Babinet's principle, the shadow produced by an optically thin Gaussian cloud will itself propagate as a Gaussian beam. If the $e^{-1}$ radius of the cloud is $r_{0}$ and the aperture has diameter $D$ located a distance $L$ from the atoms, the fraction of the shadow blocked by the aperture is

$$
e^{-\left(\pi D r_{0} / \lambda L\right)^{2}}
$$

Even for $r_{0}=10 \mu \mathrm{m}$, corresponding to a nondegenerate distribution at $50 \mathrm{nK}$, this fraction is negligible. The asymmetry evident in the halo of Fig. 1(b) is due to the asymmetry of the trap harmonic coefficients: The diffraction angle is larger in the direction of tighter confinement.
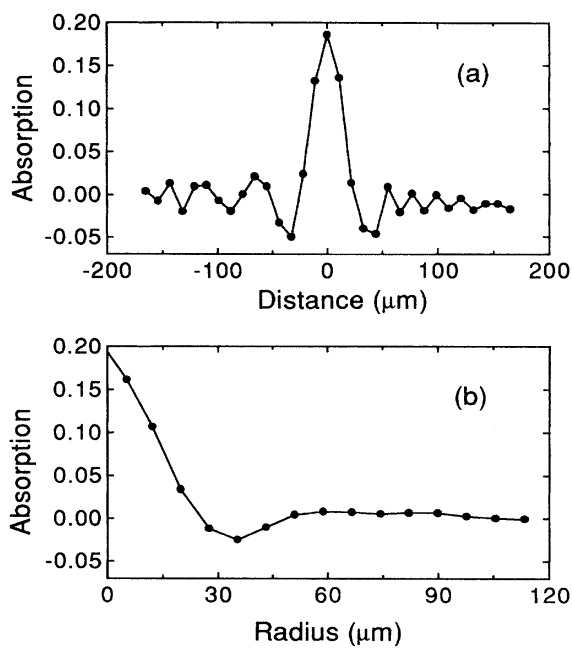

FIG. 2. Cross section (a) and angular average (b) of the absorption image shown in Fig. 1(b). The cross section in (a) is along the trap $x-y$ axis, and each solid circle corresponds to a pixel on the CCD camera. The profile in (b) is obtained by scaling the image data according to the asymmetry of the trap, and averaging around the center of the trap for each radius. The location of each solid circle corresponds to the mean radius of the annulus being averaged. The halo evident in Fig. 1(b) manifests itself as areas of negative absorption beside the peak. The probe detuning is $18 \pm 1.5 \mathrm{MHz}$ below the atomic resonance frequency.

In order to test this interpretation, the experiment was modeled numerically. A Gaussian wave front was attenuated according to the Bose-Einstein density distribution for a harmonic oscillator, and then propagated through an appropriate aperture and lens to the image plane. Because of the numerical intensity of the calculation, the beam profile was approximated as one dimensional rather than cylindrical. The results of the calculation are consistent with observation, in that diffraction features are not seen in the image plane when $N / N_{c}<1$, but appear abruptly for larger numbers. This point was verified for temperatures as low as $50 \mathrm{nK}$.

The shape and size of the diffraction features given by the model in the presence of a condensate did not agree quantitatively with the halos observed experimentally. This disagreement may be attributed to unusual light scattering properties of the dense condensate, or to the onedimensional nature of the model. However, the light scattering properties of the noncondensed part of the gas are more certain, because multiple scattering and cooperative scattering processes cannot occur. Multiple photon scattering is ruled out by the low optical absorption. The peak absorption is as low as $20 \%$ for some of the data with low values of $T$ and high values of $N / N_{c}$. Cooperative scattering, such as superfluorescence, is important when the density is such that $n \geq(2 \pi / \lambda)^{3}=8 \times 10^{14} \mathrm{~cm}^{-3}$. At $400 \mathrm{nK}$, the critical density of the noncondensed gas is only $2 \times 10^{12} \mathrm{~cm}^{-3}$, and at lower temperatures it decreases as $T^{3 / 2}$. 
While our estimates of the temperature and number are relatively accurate when $N / N_{c}<1$, the diffraction by the condensate changes both the size and shape of the absorption signal. The narrowest peaks, such as the one shown in Fig. 1(b), are not Gaussian, as can be seen in the profiles of the absorption signal shown in Fig. 2. The $e^{-1}$ radius of the peak implies a temperature of $\sim 100 \mathrm{nK}$, but, given the uncertainties in measuring small radii and the presence of diffraction effects, the temperature may be as high as $150 \mathrm{nK}$ or as low as zero. This temperature range is comparable to the trap zero-point energy of $10 \mathrm{nK}$. Similarly, the number of atoms is difficult to determine because not all of the diffracted light is imaged and the scattering properties of a Bose condensate are uncertain [25]. However, the total absorption indicates $N=2 \times$ $10^{4}$, while the peak absorption indicates $N=4 \times 10^{4}$. Five cycles resulted in images similar to Fig. 1(b), with nominal temperatures of $\sim 100 \mathrm{nK}$ and numbers from $2 \times 10^{4}$ to $2 \times 10^{5}$. At $100 \mathrm{nK}, N_{c}$ is only 3500 atoms, suggesting that a majority of the atoms reside in the condensate. The numbers of condensate atoms implied by these measurements is significantly greater than the maximum of 1500 atoms predicted for a stable condensate by mean-field theories [13], though a recent study of threebody interactions suggests that condensates with more atoms may be possible [26]. However, we caution that due to the uncertainties described above, more quantitative measurements are needed.

We have presented evidence that a gas with negative scattering length can undergo BEC. Our observations imply that the gas does not form a liquid or solid in tens of seconds that elapse between entering the quantum degenerate regime during the final stages of evaporative cooling, and the subsequent absorption image exposure. Otherwise, an abrupt loss of atoms would be observed as the latent heat from the ordinary phase transition was released. In the future, we plan to use a lower $f$-number optical system and microwave spectroscopy to more directly detect the condensate, and to quantitatively measure its occupation number, spatial size, and lifetime. Many intriguing possibilities await further exploration, including gaseous superfluidity, anomalous light scattering [25], and experiments with the fermionic atom ${ }^{6} \mathrm{Li}$.

The authors are grateful to $\mathrm{M}$. Holland for a helpful discussion. We thank Hewlett-Packard for loaning us the microwave synthesizer used in this experiment. This work is supported by the National Science Foundation and the Welch Foundation.

[1] A. Einstein, Sitzungsber. Kgl. Preuss. Akad. Wiss. 1924, 261 (1924); S. N. Bose, Z. Phys. 26, 178 (1924).

[2] J. L. Lin and J. P. Wolfe, Phys. Rev. Lett. 71, 1222 (1993).

[3] See the following reviews: T. J. Greytak and D. Kleppner, in New Trends in Atomic Physics, edited by G. Grynberg and R. Stora (North-Holland, Amsterdam, 1984), Vol. 2, p. 1125; I. F. Silvera and J. T. M. Walraven, in Progress in Low Temperature Physics, edited by D.F. Brewer
(North-Holland, Amsterdam, 1986), Vol. 10, p. 139; T. J. Greytak, in Bose Einstein Condensation, edited by A. Griffen, D. W. Snoke, and A. Stringari (Cambridge University Press, Cambridge, 1995), p. 131.

[4] M.H. Anderson, J.R. Ensher, M.R. Matthews, C.E. Wieman, and E. A. Cornell, Science 269, 198 (1995).

[5] See, for instance, K. Huang, Statistical Mechanics (John Wiley \& Sons, New York, 1987).

[6] See, for instance, C. J. Joachain, Quantum Collision Theory (Elsevier Science Publishers B.V., Amsterdam, 1975).

[7] J. R. Gardner et al., Phys. Rev. Lett. 74, 3764 (1995).

[8] A. J. Moerdijk, W. C. Stwalley, R.G. Hulet, and B. J. Verhaar, Phys. Rev. Lett. 72, 40 (1994); R. Côté, A. Dalgarno, and M. J. Jamieson, Phys. Rev. A 50, 399 (1994).

[9] E. R. I. Abraham, W. I. McAlexander, C. A. Sackett, and R. G. Hulet, Phys. Rev. Lett. 74, 1315 (1995).

[10] A. L. Fetter and J. D. Walecka, Quantum Theory of ManyParticle Systems (McGraw-Hill, New York, 1971).

[11] T.D. Lee, K. Huang, and C. N. Yang, Phys. Rev. 106, 1135 (1957).

[12] H. T. C. Stoof, Phys. Rev. A 49, 3824 (1994).

[13] P. A. Ruprecht, M. J. Holland, K. Burnett, and M. Edwards, Phys. Rev. A 51, 4704 (1995); Y. Kagan, G. V. Shlyapnikov, and J.T.M. Walraven (to be published).

[14] J. J. Tollett, C. C. Bradley, C. A. Sackett, and R. G. Hulet, Phys. Rev. A 51, R22 (1995).

[15] A.L. Migdall, J. V. Prodan, W. D. Phillips, T.H. Bergeman, and H. J. Metcalf, Phys. Rev. Lett. 54, 2596 (1985).

[16] W. Petrich, M.H. Anderson, J. R. Ensher, and E. A. Cornell, Phys. Rev. Lett. 74, 3352 (1995); K. B. Davis, M.-O. Mewes, M. A. Joffe, M. R. Andrews, and W. Ketterle, Phys. Rev. Lett. 74, 5202 (1995).

[17] The trap bias field and harmonic frequencies are different than those reported in Ref. [14]. We believe these changes can be attributed to partial demagnetization occurring during a vacuum bake.

[18] V. Bagnato, D. E. Pritchard, and D. Kleppner, Phys. Rev. A 35, 4354 (1987).

[19] H.F. Hess, Phys. Rev. B 34, 3476 (1986).

[20] N. Masuhara et al., Phys. Rev. Lett. 61, 935 (1988); J. M. Doyle et al., Phys. Rev. Lett. 67, 603 (1991); I. D. Setija et al., Phys. Rev. Lett. 70, 2257 (1993).

[21] C. S. Adams, H. J. Lee, N. Davidson, M. Kasevich, and S. Chu, Phys. Rev. Lett. 74, 3577 (1995).

[22] D.E. Pritchard, K. Helmerson, and A. G. Martin, in Proceedings of the 11th International Conference on Atomic Physics, edited by S. Haroche, J. C. Gay, and G. Grynberg (World Scientific, Singapore, 1989), p. 179.

[23] C. A. Sackett, C. C. Bradley, and R. G. Hulet (to be published).

[24] A. J. Moerdijk and B.J. Verhaar (personal communication).

[25] B. V. Svistunov and G. V. Shlyapnikov, Sov. Phys. JETP 71, 71 (1990); H.D. Politzer, Phys. Rev. A 43, 6444 (1991); J. Javanainen, Phys. Rev. Lett. 72, 2375 (1994); L. You, M. Lewenstein, and J. Cooper, Phys. Rev. A 50, R3565 (1994); O. Morice, Y. Castin, and J. Dalibard, Phys. Rev. A 51, 3896 (1995).

[26] B.D. Esry, C. H. Greene, Y. Zhou, and C. D. Lin (to be published). 


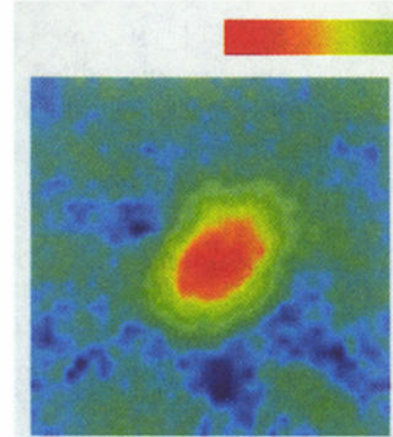

(a)

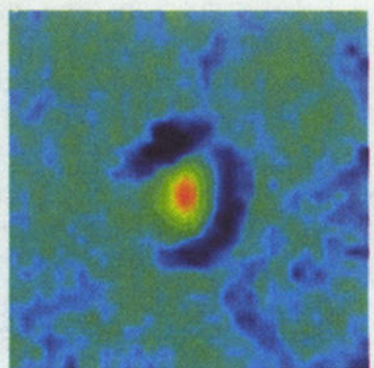

(b)

FIG. 1 (color). False-color images of trapped atom clouds resulting from different load-evaporate cycles. Images are obtained by passing a probe laser beam through the atom cloud and imaging its near-field absorption profile on a CCD camera. These images are then subtracted from a background image taken without atoms present. The spatial scale corresponds to $250 \mu \mathrm{m}$ along each axis at the location of the atoms. In these images, the diagonal extending from the lower right corner to the upper left corner is aligned with the trap $x-y$ direction. The color scale above the images maps the colors to probe absorption. The scale varies linearly from $-15 \%$ (violet) to $20 \%$ (red) absorption, where negative values indicate areas that are brighter in the presence of atoms. (a) corresponds to $1.2 \times 10^{5}$ atoms at $590 \mathrm{nK}$, and (b) to the nominal values of $2 \times 10^{4}$ atoms at $100 \mathrm{nK}$. The halo surrounding the absorption peak visible in (b) is attributed to diffraction from the spatially localized Bose-Einstein condensate. 